\title{
BEST CONSTANTS IN THE WEAK-TYPE ESTIMATES FOR UNCENTERED MAXIMAL OPERATORS
}

\author{
ADAM OSȨKOWSKI \\ Faculty of Mathematics, Informatics and Mechanics, University of Warsaw, \\ Banacha 2, 02-097 Warsaw, Poland \\ e-mail:ados@mimuw.edu.pl
}

(Received 20 November 2011; accepted 25 January 2012; first published online 30 March 2012)

\begin{abstract}
Let $\mu$ be a Borel measure on $\mathbb{R}$. The paper contains the proofs of the estimates$$
\left\|\mathcal{M}_{\mu} f\right\|_{L^{q, \infty}(A, \mu)} \leq c_{p, q}\|f\|_{L^{p}(\mathbb{R}, \mu)} \mu(A)^{1 / q-1 / p}, \quad 1 \leq p<\infty, q \in(0, p],
$$

and

$$
\left\|\mathcal{M}_{\mu} f\right\|_{L^{q, \infty}(A, \mu)} \leq C_{p, q} \mid f f \|_{L^{p, \infty}(\mathbb{R}, \mu)} \mu(A)^{1 / q-1 / p}, \quad 1<p<\infty, q \in(0, p]
$$

Here $A$ is a subset of $\mathbb{R}, f$ is a $\mu$-locally integrable function, $\mathcal{M}_{\mu}$ is the uncentred maximal operator with respect to $\mu$ and $c_{p, q}$, and $C_{p, q}$ are finite constants depending only on the parameters indicated. In the case when $\mu$ is the Lebesgue measure, the optimal choices for $c_{p, q}$ and $C_{p, q}$ are determined. As an application, we present some related tight bounds for the strong maximal operator on $\mathbb{R}^{n}$ with respect to a general product measure.
\end{abstract}

2000 Mathematics Subject Classification. Primary: 42B25. Secondary: 42B35, 46E30.

1. Introduction. Suppose $\mu$ is a non-negative Borel measure on $\mathbb{R}^{n}$ and let $f$ : $\mathbb{R}^{n} \rightarrow \mathbb{R}$ be a $\mu$-locally integrable function. The uncentred maximal function of $f$ with respect to $\mu$ is given by the formula

$$
\left(\mathcal{M}_{\mu} f\right)(x)=\sup _{x \in B} \frac{1}{\mu(B)} \int_{B}|f| \mathrm{d} \mu,
$$

where the supremum is taken over all closed balls $B$, which contain the point $x$. If $\mu$ is the Lebesgue measure, then $\mathcal{M}_{\mu}$ is the usual uncentred maximal operator of Hardy and Littlewood [4]. It is well known (see, e.g. Stein [6]) that if $\mu$ satisfies the doubling condition

$$
\mu(B(x, 2 r)) \leq C \mu(B(x, r)) \quad \text { for some } C<\infty \text { and all } x \in \mathbb{R}^{n}, r>0
$$

(here $B(x, r)$ denotes the closed ball of centre $x$ and radius $r$ ), then $\mathcal{M}_{\mu}$ maps $L^{p}\left(\mathbb{R}^{n}, \mu\right)$ into itself for $p>1$, and $L^{1}\left(\mathbb{R}^{n}, \mu\right)$ into $L^{1, \infty}\left(\mathbb{R}^{n}, \mu\right)$. This is still true, without the doubling property if and only if $n=1$ (see $[\mathbf{1}, \mathbf{2}, \mathbf{5}])$. 
The question about the precise evaluation of strong and weak norms of $\mathcal{M}_{\mu}$ has gained some interest in the literature, and the objective of this paper is to establish two new results of this type. We will be particularly interested in the one-dimensional case. We have the following $L^{p}$-estimates for $\mathcal{M}_{\mu}$ : For any $\mu$-locally integrable $f$ and $1<p<\infty$ we have

$$
\left\|\mathcal{M}_{\mu} f\right\|_{L^{p}(\mathbb{R}, \mu)} \leq c_{p}\|f\|_{L^{p}(\mathbb{R}, \mu)},
$$

where $c_{p}$ is the unique positive solution of the equation

$$
(p-1) x^{p}-p x^{p-1}-1=0 .
$$

This statement, with $\mu$ being the Lebesgue measure, was proved by Grafakos and Montgomery-Smith in [3]; for the general case, consult Grafakos and Kinnunen [2]. In general, constant $c_{p}$ in (1.1) cannot be replaced by a smaller number, see [3]. The $L^{1}$-inequality does not hold in general with any finite constant $c_{1}$, but we have the sharp weak-type estimate

$$
\left\|\mathcal{M}_{\mu} f\right\|_{L^{1, \infty}(\mathbb{R}, \mu)} \leq 2|| f \|_{L^{1}(\mathbb{R}, \mu)}
$$

as proved in [2]. Here, as usual, for any Borel subset $A$ of $\mathbb{R}$ and any $0<p<\infty$, we define the weak $p$-th norm of $f$ on $A$ by the formula

$$
\|f\|_{L^{p, \infty}(A, \mu)}=\sup _{\lambda>0} \lambda[\mu(\{x \in A:|f(x)|>\lambda\})]^{1 / p} .
$$

There is a natural question about the best constants in the corresponding weak-type $(p, p)$ estimates for $\mathcal{M}_{\mu}, 1<p<\infty$. In fact, we will study this question in a more general setting and compare the weak $q$-th norm of $\mathcal{M}_{\mu} f$ to the $p$-th norm of $f$, where $p \geq 1$ and $q \in(0, p]$. Introduce constant

$$
C_{p}=\frac{(p-1)\left(2^{p /(p-1)}-1\right)}{p}\left((p-1)\left(2^{p /(p-1)}-2\right)\right)^{-1 / p}
$$

when $1<p<\infty$, and put $C_{1}=2$. We will establish the following result.

THEOREM 1.1. For any $\mu$-locally integrable function $f: \mathbb{R} \rightarrow \mathbb{R}$, any Borel subset $A$ of $\mathbb{R}$ and any $1 \leq p<\infty, q \in(0, p]$, we have

$$
\left\|\mathcal{M}_{\mu} f\right\|_{L^{q, \infty}(A, \mu)} \leq C_{p}\|f\|_{L^{p}(\mathbb{R}, \mu)} \mu(A)^{1 / q-1 / p} .
$$

If $\mu$ is the Lebesgue measure, then the constant $C_{p}$ is the best possible.

In particular, if $p=q$, then (1.3) yields the weak-type $(p, p)$ estimate

$$
\left\|\mathcal{M}_{\mu} f\right\|_{L^{p, \infty}(\mathbb{R}, \mu)} \leq C_{p}\|f\|_{L^{p}(\mathbb{R}, \mu)}
$$

which, as we will see, is also sharp, provided $\mu$ is the Lebesgue measure.

The next problem we will study concerns the sharp comparison of the weak norms of $f$ and $\mathcal{M}_{\mu} f$. Here constants $c_{p}$ of Grafakos and Montgomery-Smith [3] come into play; we will prove the following statement. 
THEOREM 1.2. For any $\mu$-locally integrable function $f: \mathbb{R} \rightarrow \mathbb{R}$, any Borel subset $A$ of $\mathbb{R}$ and any $1<p<\infty, q \in(0, p]$, we have

$$
\left\|\mathcal{M}_{\mu} f\right\|_{L^{q, \infty}(A, \mu)} \leq c_{p}\|f\|_{L^{p, \infty}(\mathbb{R}, \mu)} \mu(A)^{1 / q-1 / p} .
$$

If $\mu$ is the Lebesgue measure, then the constant $c_{p}$ is the best possible.

As previously, let us distinguish the choice $p=q \in(1, \infty)$. It gives the bound

$$
\left\|\mathcal{M}_{\mu} f\right\|_{L^{p, \infty}(\mathbb{R}, \mu)} \leq c_{p}\|f\|_{L^{p, \infty}(\mathbb{R}, \mu)},
$$

which will be proved to be sharp in the case when $\mu$ is the Lebesgue measure.

Theorems 1.1 and 1.2 will be established in the next section. In Section 3 we will apply these two theorems to obtain related results in the higher dimensional setting: more precisely, we will show tight weak-type estimates for the so-called strong maximal operator on $\mathbb{R}^{n}, n \geq 2$.

2. Proofs of theorems 1.1 and 1.2. We start with recalling the main lemma from [2] (see also [3] for the special case in which $\mu$ is the Lebesgue measure). This result can be regarded as an appropriate version of the weak-type estimate for $\mathcal{M}_{\mu}$. Here and below, we use the notation $\{f>\lambda\}$ for the set $\{x \in \mathbb{R}$ : $f(x)>\lambda\}$.

LEMMA 2.1. If $f$ is a non-negative and $\mu$-locally integrable function on $\mathbb{R}$, then for any $\lambda>0$ we have

$$
\lambda\left(\mu\left(\left\{\mathcal{M}_{\mu} f>\lambda\right\}\right)+\mu(\{f>\lambda\})\right) \leq \int_{\left\{\mathcal{M}_{\mu} f>\lambda\right\}} f d \mu+\int_{\{f>\lambda\}} f d \mu .
$$

In other words, for any $f, \lambda$ as in the statement above, we have

$$
\int_{\mathbb{R}} u\left(f(x) / \lambda, \mathcal{M}_{\mu} f(x) / \lambda\right) \mathrm{d} \mu(x) \leq 0,
$$

where $u:[0, \infty) \times[0, \infty] \rightarrow \mathbb{R}$ is the function given by the formula

$$
u(x, y)=\left(\chi_{\{x>1\}}+\chi_{\{y>1\}}\right)(1-x) .
$$

Introduce the parameters

$$
r_{p}=\frac{p}{(p-1)\left(2^{p /(p-1)}-1\right)}, \quad s_{p}=\frac{p 2^{1 /(p-1)}}{(p-1)\left(2^{p /(p-1)}-1\right)}
$$

and

$$
\alpha_{p}=\frac{2^{p /(p-1)}-1}{2^{p /(p-1)}-2}
$$

LEMMA 2.2. For any $0 \leq x \leq y$ and any $1<p<\infty$, we have

$$
\alpha_{p} u(x, y) \geq \chi_{\{y>1\}}-C_{p}^{p} x^{p} .
$$


Proof. If $y \leq 1$, then the estimate becomes $0 \geq-C_{p}^{p} x^{p}$, which is obvious. Suppose $y>1$ and $x \leq 1$. Then (2.3) is equivalent to

$$
F(x):=\alpha_{p}(1-x)-1+C_{p}^{p} x^{p} \geq 0,
$$

which holds true for all $x \geq 0$. This is the consequence of the fact that $F$ is a convex function, combined with equalities $F\left(r_{p}\right)=F^{\prime}\left(r_{p}\right)=0$. Finally, if both $x$ and $y$ are larger than 1 , inequality (2.3) can be rewritten in the form

$$
G(x):=2 \alpha_{p}(1-x)-1+C_{p}^{p} x^{p} \geq 0,
$$

which follows from the convexity of $G$ and equalities $G\left(s_{p}\right)=G^{\prime}\left(s_{p}\right)=0$.

Proof of (1.3) We may assume that $f$ is a non-negative function which satisfies $\|f\|_{L^{p}(\mathbb{R}, \mu)}<\infty$. Combining (2.2) and (2.3), we obtain that for $p>1$,

$$
\lambda^{p} \mu\left(\left\{\mathcal{M}_{\mu} f>\lambda\right\}\right) \leq C_{p}^{p}|| f \|_{L^{p}(\mathbb{R}, \mu)}^{p} .
$$

This bound is also true for $p=1$, as we have already mentioned above. Thus, since $\mu\left(\left\{x \in A: \mathcal{M}_{\mu} f(x)>\lambda\right\}\right) \leq \min \left\{\mu(A), \mu\left(\left\{\mathcal{M}_{\mu} f>\lambda\right\}\right)\right\}$, we have

$$
\begin{aligned}
\lambda^{q} \mu\left(\left\{x \in A: \mathcal{M}_{\mu} f(x)>\lambda\right\}\right) & \leq \lambda^{q} \mu\left(\left\{\mathcal{M}_{\mu} f \geq \lambda\right\}\right)^{q / p} \mu(A)^{1-q / p} \\
& \leq C_{p}^{q}\|f\|_{L^{p}(\mathbb{R}, \mu)}^{q} \mu(A)^{1-q / p}
\end{aligned}
$$

where the latter passage is due to (2.4). It remains to take supremum over $\lambda$ in (2.5) to obtain (1.3).

Sharpness for the Lebesgue measure. Let $r_{p}$ and $s_{p}$ be as above and introduce the parameter $\beta_{p}=2\left(s_{p}-1\right) /\left(1-r_{p}\right)$. Consider the function

$$
f=s_{p} \chi_{[-1,1]}+r_{p}\left(\chi_{\left[-\beta_{p}-1,-1\right)}+\chi_{\left(1, \beta_{p}+1\right]}\right)
$$

and let $A=\left[-\beta_{p}-1, \beta_{p}+1\right]$. The identity

$$
\frac{1}{\left|\left[-\beta_{p}-1,1\right]\right|} \int_{-\beta_{p}-1}^{1} f(x) \mathrm{d} x=\frac{1}{\left|\left[-1, \beta_{p}+1\right]\right|} \int_{-1}^{\beta_{p}+1} f(x) \mathrm{d} x=\frac{2 s_{p}+\beta_{p} r_{p}}{2+\beta_{p}}=1
$$

and the definition of the maximal operator imply that $\mathcal{M}_{|\cdot|} f(x) \geq 1$ for $x \in A$. Therefore,

$$
\frac{\left|\left\{x \in A: \mathcal{M}_{|\cdot|} f(x) \geq 1\right\}\right|}{\|f\|_{L^{p}(\mathbb{R},|\cdot|)}^{q}|A|^{1-q / p}}=\left(\frac{|A|}{\|f\|_{L^{p}(\mathbb{R},|\cdot|)}^{p}}\right)^{q / p}=\left(\frac{2\left(\beta_{p}+1\right)}{2 \beta_{p} r_{p}^{p}+2 s_{p}^{p}}\right)^{q / p},
$$

and the latter expression is easily checked to be equal to $C_{p}^{q}$. This proves the sharpness of (1.3). The same example yields the optimality of $C_{p}$ in (1.4): we have

$$
\left\|\mathcal{M}_{|\cdot|} f\right\|_{L^{p, \infty}(\mathbb{R},|\cdot|)}^{p} \geq\left|\left\{\mathcal{M}_{|\cdot|} f \geq 1\right\}\right| \geq|A|=C_{p}^{p}|| f \|_{L^{p}(\mathbb{R},|\cdot|)}^{p} .
$$

Proof of (1.5) It suffices to consider functions $f$, which are non-negative and satisfy $0<\|f\|_{L^{p, \infty}(\mathbb{R}, \mu)}<\infty$. In addition, by homogeneity, we may and do assume 
that $\|f\|_{L^{p, \infty}(\mathbb{R}, \mu)}=1$. Rewrite (2.1) in the form

$$
\lambda \mu\left(\left\{\mathcal{M}_{\mu} f>\lambda\right\}\right) \leq \int_{\left\{\mathcal{M}_{\mu} f>\lambda\right\}} f \mathrm{~d} \mu+\int_{\{f>\lambda\}}(f-\lambda) \mathrm{d} \mu .
$$

The well-known inequality of Hardy and Littlewood (see, e.g. [4]) states that if $h$ is a non-negative function and $A$ is a Borel subset of $\mathbb{R}$, then

$$
\int_{A} h \mathrm{~d} \mu \leq \int_{0}^{\mu(A)} h^{*}(t) \mathrm{d} t
$$

where $h^{*}(t)=\inf \{s>0: \mu(\{f>s\}) \leq t\}$ is the non-increasing rearrangement of $h$. Since $\|f\|_{L^{p, \infty}(\mathbb{R}, \mu)}=1$, we have $\mu(\{f>\lambda\}) \leq \lambda^{-p}$ for all $\lambda>0$ and hence $f^{*}(t) \leq t^{-1 / p}$ for all positive $t$. Putting all these facts together, we obtain

$$
\begin{aligned}
\lambda \mu\left(\left\{\mathcal{M}_{\mu} f>\lambda\right\}\right) & \leq \int_{0}^{\mu\left(\left\{\mathcal{M}_{\mu} f>\lambda\right\}\right)} t^{-1 / p} \mathrm{~d} t+\int_{0}^{\lambda^{-p}}\left(t^{-1 / p}-\lambda\right) \mathrm{d} t \\
& =\frac{p}{p-1} \mu\left(\left\{\mathcal{M}_{\mu} f>\lambda\right\}\right)^{(p-1) / p}+\frac{\lambda^{1-p}}{p-1} .
\end{aligned}
$$

Multiplying both sides by $(p-1) \lambda^{p-1}$ yields

$$
(p-1) \lambda^{p} \mu\left(\left\{\mathcal{M}_{\mu} f>\lambda\right\}\right) \leq p\left(\lambda^{p} \mu\left(\left\{\mathcal{M}_{\mu} f>\lambda\right\}\right)\right)^{(p-1) / p}+1 .
$$

In view of (1.2), this implies

$$
\lambda^{p} \mu\left(\left\{\mathcal{M}_{\mu} f>\lambda\right\}\right) \leq c_{p}^{p}=c_{p}^{p} \mid\|f\|_{L^{p, \infty}(\mathbb{R}, \mu)} .
$$

Indeed, we have $c_{p} \geq 1$ and the function $x \mapsto(p-1) x^{p}-p x^{p-1}$ is increasing on $[1, \infty)$. Thus, we have established (1.6). Furthermore, (2.7) yields

$$
\lambda^{q} \mu\left(\left\{x \in A: \mathcal{M}_{\mu} f(x)>\lambda\right\}\right) \leq c_{p}^{q} \mid\|f\|_{L^{p, \infty}(\mathbb{R}, \mu)}^{q} \mu(A)^{1-q / p},
$$

which can be seen by repeating the argument leading from (2.4) to (2.5). The proof of (1.5) is complete.

Sharpness for the Lebesgue measure. Fix $p>1$ and let $f: \mathbb{R} \rightarrow \mathbb{R}$ be given by $f(t)=|2 t|^{-1 / p}$. It is easy to check that $\|f\|_{L^{p, \infty}(\mathbb{R})}=1$. Furthermore, for any $x>0$ we have

$$
\frac{1}{\left|\left[-c_{p}^{-p} x, x\right]\right|} \int_{-c_{p}^{p} x}^{x} f(t) \mathrm{d} t=(2 x)^{-1 / p} \frac{p\left(1+c_{p}^{1-p}\right)}{(p-1)\left(1+c_{p}^{-p}\right)}=c_{p}(2 x)^{-1 / p},
$$

where the latter equality follows from (1.2). Thus, by the definition of the maximal operator, we have $\mathcal{M}_{|\cdot|} f(x) \geq c_{p}(2 x)^{-1 / p}$ for $x>0$ and similarly $\mathcal{M}_{|\cdot|} f(x) \geq c_{p}(-2 x)^{-1 / p}$ for negative $x$. Consequently, $\left\|\mathcal{M}_{|\cdot|} f\right\|_{L^{p, \infty}(\mathbb{R},|\cdot|)} \geq c_{p}$ and the equality in (1.6) is attained. Next, putting $A=\left\{\mathcal{M}_{|\cdot|} f \geq 1\right\}$, we see that $\left[-c_{p}^{p} / 2, c_{p}^{p} / 2\right] \subseteq A$ and hence

$$
\left\|\mathcal{M}_{|\cdot|} f\right\|_{L^{q, \infty}(A,|\cdot|)}^{q} \geq|A| \geq c_{p}^{q}|A|^{1-q / p}=c_{p}^{q}|A|^{1-q / p}|| f \|_{L^{p, \infty}(\mathbb{R},|\cdot|)}^{q} .
$$

This yields the desired optimality of $c_{p}$ in (1.5). 
3. Estimates for the strong maximal function. This section contains applications of previous results to the study of maximal operators in higher dimensions. Let $n \geq 1$ be a fixed integer and let $\mu$ be a product measure on $\mathbb{R}^{n}: \mu=\mu_{1} \otimes \mu_{2} \otimes \ldots \otimes \mu_{n}$ for some Borel measures $\mu_{1}, \mu_{2}, \ldots, \mu_{n}$ on $\mathbb{R}$. The strong maximal operator $M_{\mu}$ is an operator that acts on $\mu$-locally integrable functions $f$ by the formula

$$
M_{\mu} f(x)=\sup _{x \in D} \frac{1}{\mu(D)} \int_{D}|f| \mathrm{d} \mu
$$

where the supremum is taken over all closed rectangles $D$, with sides parallel to the axes, satisfying $x \in D$. Observe that for $n=1$, operators $M_{\mu}$ and $\mathcal{M}_{\mu}$ coincide.

We will prove the following fact.

THEOREM 3.1. Let $\mu$ and $M_{\mu}$ be as above.

(i) If $n \geq 2$, then in general $M_{\mu}$ does not map $L^{1}\left(\mathbb{R}^{n}, \mu\right)$ into $L^{1, \infty}\left(\mathbb{R}^{n}, \mu\right)$.

(ii) If $1<p<\infty$, then for any $f: \mathbb{R}^{n} \rightarrow \mathbb{R}$ we have

$$
\left\|M_{\mu} f\right\|_{L^{p, \infty}\left(\mathbb{R}^{n}, \mu\right)} \leq C_{p} c_{p}^{n-1}|| f \|_{L^{p}\left(\mathbb{R}^{n} \mu\right)} .
$$

If $\mu$ is the Lebesgue measure on $\mathbb{R}^{n}$, then the constant has the optimal order $O\left((p-1)^{1-n}\right)$ as $p \rightarrow 1$.

(iii) If $1<p<\infty$, then for any $f: \mathbb{R}^{n} \rightarrow \mathbb{R}$ we have

$$
\left\|M_{\mu} f\right\|_{L^{p, \infty}\left(\mathbb{R}^{n}, \mu\right)} \leq c_{p}^{n} \mid f \|_{L^{p, \infty}\left(\mathbb{R}^{n}, \mu\right)} .
$$

If $\mu$ is the Lebesgue measure on $\mathbb{R}^{n}$, then the constant is the best possible.

REMARK 3.2. By the argument from the previous section, (3.1) and (3.2) imply the estimates

$$
\left\|M_{\mu} f\right\|_{L^{q, \infty}(A, \mu)} \leq C_{p} c_{p}^{n-1}|| f \|_{L^{p}\left(\mathbb{R}^{n} \mu\right)} \mu(A)^{1 / q-1 / p}
$$

and

$$
\left\|M_{\mu} f\right\|_{L^{q, \infty}(A, \mu)} \leq c_{p}^{n}|| f \|_{L^{p, \infty}\left(\mathbb{R}^{n}, \mu\right)} \mu(A)^{1 / q-1 / p}
$$

for all $\mu$-locally integrable functions $f: \mathbb{R}^{n} \rightarrow \mathbb{R}$, all Borel subsets $A$ of $\mathbb{R}^{n}$ and all $1<p<\infty, 0<q \leq p$. We will prove below that (3.3) is sharp, provided $\mu$ is the Lebesgue measure.

Proof of Theorem 3.1. (i) This will be shown in the proof of (ii) below.

(ii) The key observation is that

$$
M_{\mu} \leq \mathcal{M}_{\mu_{1}}^{(1)} \circ \mathcal{M}_{\mu_{2}}^{(2)} \circ \ldots \circ \mathcal{M}_{\mu_{n}}^{(n)}
$$

where $\mathcal{M}_{\mu_{k}}^{(k)}$ denotes the maximal operator $\mathcal{M}_{\mu_{k}}$ applied to the $k$-th coordinate. Let $f$ be a non-negative function on $\mathbb{R}^{n}$ satisfying $\|f\|_{L^{p}\left(\mathbb{R}^{n}, \mu\right)}<\infty$. Using (1.4) with respect 
to $\mathcal{M}_{\mu_{1}}$ and then (1.1) with respect to $\mathcal{M}_{\mu_{2}}, \mathcal{M}_{\mu_{3}}, \ldots, \mathcal{M}_{\mu_{n}}$, we obtain

$$
\begin{aligned}
\lambda^{p} & \mu\left(\left\{\mathcal{M}_{\mu_{1}}^{(1)} \circ \mathcal{M}_{\mu_{2}}^{(2)} \circ \ldots \circ \mathcal{M}_{\mu_{n}}^{(n)} f>\lambda\right\}\right) \\
& =\int_{\mathbb{R}^{n-1}} \lambda^{p} \mu_{1}\left(\left\{x_{1}: \mathcal{M}_{\mu_{1}}^{(1)} \circ \ldots \circ \mathcal{M}_{\mu_{n}}^{(n)} f\left(x_{1}, x_{2}, \ldots, x_{n}\right)>\lambda\right\}\right) \mathrm{d} \mu_{2}\left(x_{2}\right) \ldots \mathrm{d} \mu_{n}\left(x_{n}\right) \\
& \leq C_{p}^{p} \int_{\mathbb{R}^{n-1}}\left[\int_{\mathbb{R}}\left[\mathcal{M}_{\mu_{2}}^{(2)} \circ \ldots \circ \mathcal{M}_{\mu_{n}}^{(n)} f\left(x_{1}, x_{2}, \ldots, x_{n}\right)\right]^{p} \mathrm{~d} \mu_{1}\left(x_{1}\right)\right] \mathrm{d} \mu_{2}\left(x_{2}\right) \ldots \mathrm{d} \mu_{n}\left(x_{n}\right) \\
& =C_{p}^{p} \int_{\mathbb{R}^{n}}\left[\mathcal{M}_{\mu_{2}}^{(2)} \circ \ldots \circ \mathcal{M}_{\mu_{n}}^{(n)} f\left(x_{1}, x_{2}, \ldots, x_{n}\right)\right]^{p} \mathrm{~d} \mu_{1}\left(x_{1}\right) \mathrm{d} \mu_{2}\left(x_{2}\right) \ldots \mathrm{d} \mu_{n}\left(x_{n}\right) \\
& =C_{p}^{p} \int_{\mathbb{R}^{n-1}}\left[\int_{\mathbb{R}}\left[\mathcal{M}_{\mu_{2}}^{(2)} \circ \ldots \circ \mathcal{M}_{\mu_{n}}^{(n)} f(x)\right]^{p} \mathrm{~d} \mu_{2}\left(x_{2}\right)\right] \mathrm{d} \mu_{1}\left(x_{1}\right) \mathrm{d} \mu_{3}\left(x_{3}\right) \ldots \mathrm{d} \mu_{n}\left(x_{n}\right) \\
& \leq C_{p}^{p} c_{p}^{p} \int_{\mathbb{R}^{n}}\left[\mathcal{M}_{\mu_{3}}^{(3)} \circ \ldots \circ \mathcal{M}_{\mu_{n}}^{(n)} f\left(x_{1}, x_{2}, \ldots, x_{n}\right)\right]^{p} \mathrm{~d} \mu_{1}\left(x_{1}\right) \mathrm{d} \mu_{2}\left(x_{2}\right) \ldots \mathrm{d} \mu_{n}\left(x_{n}\right) \\
& \leq \ldots \\
& \leq C_{p}^{p} c_{p}^{(n-1) p}|| f \|_{L^{p}\left(\mathbb{R}^{n}, \mu\right)}^{p} .
\end{aligned}
$$

This yields (3.1). It is not difficult to check that $1 \leq C_{p} \leq 2$ and $\frac{p}{p-1} \leq c_{p} \leq \frac{2 p}{p-1}$ for $1<p<\infty$, so the constant $C_{p} c_{p}^{n-1}$ is of the order $O\left((p-1)^{1-n}\right)$ when $p \rightarrow 1$. To see that this order is optimal when $\mu$ is the Lebesgue measure, take $p \in(1,2), n \geq 2$ and put $f=\chi_{[-1,1]^{n}}$. Then, for any $x=\left(x_{1}, x_{2}, \ldots, x_{n}\right) \in \mathbb{R}^{n}$, we have

$$
M_{\mu} f(x) \geq \prod_{k=1}^{n} \min \left(\frac{2}{\left|x_{k}\right|+1}, 1\right),
$$

which can be verified by considering the smallest rectangle that contains $x$ and the cube $[-1,1]^{n}$. Thus, for any $\lambda \in(0,1)$ we may write

$$
\begin{aligned}
\left|\left\{M_{\mu} f>\lambda\right\}\right| & \geq 2^{n}\left|\left\{x \in[1, \infty)^{n}: \prod_{k=1}^{n} \frac{2}{x_{k}+1}>\lambda\right\}\right| \\
& =2^{n} \int_{1}^{a_{1}} \int_{1}^{a_{2}} \ldots \int_{1}^{a_{n}} \mathrm{~d} x_{n} \mathrm{~d} x_{n-1} \ldots \mathrm{d} x_{1},
\end{aligned}
$$

where $a_{1}=2 / \lambda-1$ and

$$
a_{k}=\frac{2^{k}}{\lambda\left(x_{1}+1\right) \ldots\left(x_{k-1}+1\right)}-1, \quad k=2,3, \ldots, n .
$$

Denote the right-hand side of (3.5) by $\gamma_{n}$. Deriving the inner integral with respect to $x_{n}$ gives the identity

$$
\gamma_{n}=2^{n} \int_{1}^{a_{1}} \int_{1}^{a_{2}} \ldots \int_{1}^{a_{n-1}} \frac{2^{n}}{\lambda\left(x_{1}+1\right) \ldots\left(x_{n-1}+1\right)} \mathrm{d} x_{n-1} \ldots \mathrm{d} x_{1}-4 \gamma_{n-1},
$$

valid for $n \geq 2$. By induction, we easily verify that

$$
\int_{1}^{a_{k}} \ldots \int_{1}^{a_{n-1}} \frac{1}{\left(x_{k}+1\right) \ldots\left(x_{n-1}+1\right)} \mathrm{d} x_{n-1} \ldots \mathrm{d} x_{k}=\frac{1}{(n-k) !}\left(\log \frac{a_{k}+1}{2}\right)^{n-k}
$$


and hence

$$
\frac{\gamma_{n}}{4^{n}}=\frac{\left(\log \lambda^{-1}\right)^{n-1}}{\lambda(n-1) !}-\frac{\gamma_{n-1}}{4^{n-1}}
$$

This, in turn, implies that for $n \geq 3$,

$$
\frac{\gamma_{n}}{4^{n}}=\frac{\left(\log \lambda^{-1}\right)^{n-1}}{\lambda(n-1) !}-\frac{\left(\log \lambda^{-1}\right)^{n-2}}{\lambda(n-2) !}+\frac{\gamma_{n-2}}{4^{n-2}}>\frac{\left(\log \lambda^{-1}\right)^{n-1}}{\lambda(n-1) !}-\frac{\left(\log \lambda^{-1}\right)^{n-2}}{\lambda(n-2) !}
$$

This is also true for $n=2$ : we have $\gamma_{1}=4\left(\lambda^{-1}-1\right)$ and hence by (3.6),

$$
\frac{\gamma_{2}}{4}=\frac{\log \lambda^{-1}}{\lambda}-\frac{1}{\lambda}+1
$$

Consequently, we have $\lim _{\lambda \rightarrow 0} \lambda\left|\left\{M_{\mu} f>\lambda\right\}\right|=\infty$ and (i) is proved. Next, if we plug $\lambda=\exp (-(n-1) /(p-1))$ into $(3.7)$, we obtain that

$$
\begin{aligned}
\frac{\left\|M_{\mu} f\right\|_{L^{p, \infty}\left(\mathbb{R}^{n},|\cdot|\right)}^{p}}{\|f\|_{L^{p}\left(\mathbb{R}^{n},|\cdot|\right)}^{p}} & \geq \frac{\lambda^{p}\left|\left\{M_{\mu} f>\lambda\right\}\right|}{2^{n}} \\
& >2^{n} e^{1-n} \frac{(n-1)^{n-1}}{(n-1) !} \frac{2-p}{(p-1)^{n-1}} \\
& \geq \frac{\kappa_{n}}{(p-1)^{(n-1) p}},
\end{aligned}
$$

for some constant $\kappa_{n}$ depending only on $n$. This gives the optimality of the order.

(iii) Introduce the operators $T_{k}=\mathcal{M}_{\mu_{k}}^{(k)} \circ \mathcal{M}_{\mu_{k+1}}^{(k+1)} \circ \ldots \circ \mathcal{M}_{\mu_{n}}^{(n)}, k=1,2, \ldots, n$, and let $T_{n+1}=\mathrm{Id}$. We will prove that

$$
\left\|T_{k} f\right\|_{L^{p, \infty}(\mathbb{R}, \mu)} \leq c_{p}\left\|T_{k+1} f\right\|_{L^{p, \infty}\left(\mathbb{R}^{n} \mu\right)}
$$

for any $f$ and any $k \in\{1,2, \ldots, n\}$; this will immediately yield (3.2). To do this, fix $\lambda>0$ and let $A_{\lambda}=\left\{T_{k} f>\lambda\right\}$ and $B_{\lambda}=\left\{T_{k+1} f>\lambda\right\}$. Let $\mu^{(k)}$ denote the product measure $\mu_{1} \otimes \mu_{2} \otimes \ldots \otimes \mu_{k-1} \otimes \mu_{k+1} \otimes \ldots \otimes \mu_{n}$ on $\mathbb{R}^{n-1}$. By (2.1), applied to $\mathcal{M}_{\mu_{k}}^{(k)}$, the measure $\mu_{k}$ and the function $t \mapsto T_{k+1} f\left(x_{1}, \ldots, x_{k-1}, t, x_{k+1}, \ldots, x_{n}\right), t \in \mathbb{R}$,

$$
\begin{aligned}
& \lambda \mu_{k}\left(\left\{x_{k} \in \mathbb{R}: T_{k} f\left(x_{1}, x_{2}, \ldots, x_{n}\right)>\lambda\right\}\right) \\
& \leq \int_{\left\{x_{k} \in \mathbb{R}: T_{k} f(x)>\lambda\right\}} T_{k+1} f(x) \mathrm{d} \mu_{k}\left(x_{k}\right)+\int_{\left\{x_{k} \in \mathbb{R}: T_{k+1} f(x)>\lambda\right\}}\left(T_{k+1} f(x)-\lambda\right) \mathrm{d} \mu_{k}\left(x_{k}\right) .
\end{aligned}
$$

Integrating this over $\mathbb{R}^{n-1}$ with respect to $\mathrm{d} \mu^{(k)}\left(x_{1}, x_{2}, \ldots, x_{k-1}, x_{k+1}, \ldots, x_{n}\right)$ and multiplying both sides by $\lambda^{p-1}$, we obtain

$$
\lambda^{p} \mu\left(A_{\lambda}\right) \leq \lambda^{p-1}\left[\int_{A_{\lambda}} T_{k+1} f(x) \mathrm{d} \mu(x)+\int_{B_{\lambda}}\left(T_{k+1} f(x)-\lambda\right) \mathrm{d} \mu(x)\right] .
$$

Let $\left(T_{k+1} f\right)^{*}$ be the non-increasing rearrangement of $T_{k+1} f$ (the definition is analogous to that of one-dimensional setting). We have

$$
\mu\left(B_{\lambda}\right)=\mu\left(\left\{T_{k+1} f>\lambda\right\}\right) \leq \lambda^{-p}\left\|T_{k+1} f\right\|_{L^{p, \infty}\left(\mathbb{R}^{n}, \mu\right)}^{p},
$$


so $\left(T_{k+1} f\right)^{*}(t) \leq t^{-1 / p}\left\|T_{k+1} f\right\|_{L^{p, \infty}\left(\mathbb{R}^{n}, \mu\right)}$ for any $t>0$. Therefore, using the version of inequality (2.6) in $\mathbb{R}^{n}$, we obtain

$$
\begin{aligned}
\lambda^{p} \mu\left(A_{\lambda}\right) \leq & \lambda^{p-1}\left[\int_{0}^{\mu\left(A_{\lambda}\right)} t^{-1 / p}\left\|T_{k+1} f\right\|_{L^{p, \infty}\left(\mathbb{R}^{n}, \mu\right)} \mathrm{d} t\right. \\
& \left.+\int_{0}^{\mu\left(B_{\lambda}\right)}\left(t^{-1 / p}\left\|T_{k+1} f\right\|_{L^{p, \infty}\left(\mathbb{R}^{n}, \mu\right)}-\lambda\right) \mathrm{d} t\right] .
\end{aligned}
$$

If we apply (3.9) and compute the integrals above, we obtain an inequality which can be rewritten in the equivalent form

$$
(p-1) \frac{\lambda^{p} \mu\left(A_{\lambda}\right)}{\left\|T_{k+1} f\right\|_{L^{p, \infty}\left(\mathbb{R}^{n}, \mu\right)}^{p}} \leq p\left(\frac{\lambda^{p} \mu\left(A_{\lambda}\right)}{\left\|T_{k+1} f\right\|_{L^{p, \infty}\left(\mathbb{R}^{n}, \mu\right)}^{p}}\right)^{1-1 / p}+1 .
$$

By virtue of (1.2), this yields $\lambda^{p} \mu\left(A_{\lambda}\right) \leq c_{p}\left\|T_{k+1} f\right\|_{L^{p, \infty}\left(\mathbb{R}^{n}, \mu\right)}$ and (3.8) follows. We turn to the sharpness. Let $\mu=|\cdot|$ be the Lebesgue measure on $\mathbb{R}^{n}$, fix $p^{\prime}>p$ and consider the function

$$
f\left(x_{1}, x_{2}, \ldots, x_{n}\right)=\prod_{k=1}^{n}\left|2 x_{k}\right|^{-1 / p^{\prime}} \chi_{[-1,1]^{n}}(x) .
$$

It belongs to $L^{p}\left(\mathbb{R}^{n},|\cdot|\right)$, so in particular $\|f\|_{L^{p, \infty}\left(\mathbb{R}^{n},|\cdot|\right)}<\infty$. By $(2.8)$, applied to each coordinate (here we use the product structure of $f$ ), we have $M_{|\cdot|} f \geq c_{p}^{n} f$ on $\mathbb{R}^{n}$. Therefore, $\left\|M_{|\cdot|} f\right\|_{L^{p, \infty}\left(\mathbb{R}^{n},|\cdot|\right)} \geq c_{p^{\prime}}^{n}\|f\|_{L^{p, \infty}\left(\mathbb{R}^{n},|\cdot|\right)}$ and it remains to let $p^{\prime} \rightarrow p$ to see that $c_{p}^{n}$ is optimal in (3.2). Finally, to prove the sharpness of (3.3), let $f$ be as above. Fix $\kappa>1$ and choose $\lambda>0$ such that $\lambda^{p}|\{f>\lambda\}| \cdot \kappa>\|f\|_{L^{p, \infty}\left(\mathbb{R}^{n}, \mu\right)}^{p}$. If we put $A=\{f>\lambda\}$, then $M_{|\cdot|} f>c_{p^{\prime}}^{n} \lambda$ on $A$, so

$$
\frac{\left\|M_{|\cdot|} f\right\|_{L^{q, \infty}(A,|\cdot|)}}{\|f\|_{L^{p, \infty}\left(\mathbb{R}^{n},|\cdot|\right)}} \geq \frac{c_{p^{\prime}}^{n} \lambda|A|^{1 / q}}{\kappa^{1 / p} \lambda|A|^{1 / p}}=\frac{c_{p^{\prime}}^{n}}{\kappa}|A|^{1 / q-1 / p} .
$$

Since $\kappa>1$ and $p^{\prime}>p$ were arbitrary, constant $c_{p}^{n}$ is the best in (3.3).

ACKNOWLEDGEMENTS. The research was partially supported by MNiSW Grant N N201 397437.

\section{REFERENCES}

1. A. Bernal, A note on the one-dimensional maximal function, Proc. Roy. Soc. Edinburgh 111 A (1989), 325-328.

2. L. Grafakos and J. Kinnunen, Sharp inequalities for maximal functions associated with general measures, Proc. Roy. Soc. Edinburgh 128A (1998), 717-723.

3. L. Grafakos and S. Montgomery-Smith, Best constants for uncentered maximal functions, Bull. London Math. Soc. 29 (1997), 60-64.

4. G. H. Hardy, J. E. Littlewood and G. Pólya, Inequalities, 2nd ed. (Cambridge University Press, Cambridge, UK, 1952).

5. P. Sjögren, A remark on the maximal function for measures in $\mathbb{R}^{n}$, Amer. J. Math. $\mathbf{1 0 5}$ (1983), 1231-1233.

6. E. M. Stein, Harmonic analysis: Real variable theory, orthogonality, and oscillatory integrals (Princeton University Press, Princeton, NJ, 1993). 